\title{
Convergent and asymptotic methods for second-order difference equations with a large parameter
}

\author{
Chelo Ferreira ${ }^{1}$, José L. López ${ }^{2}$ and Ester Pérez Sinusía ${ }^{1}$ \\ ${ }^{1}$ Dpto. de Matemática Aplicada, IUMA, Universidad de Zaragoza, Zaragoza \\ e-mail: cferrei@unizar.es, ester.perez@unizar.es \\ ${ }^{2}$ Dpto. de Ingeniería Matemática e Informática, Universidad Pública de Navarra and INAMAT, Pamplona \\ e-mail: jl.lopez@unavarra.es
}

\begin{abstract}
We consider the second-order linear difference equation $y(n+2)-2 a y(n+1)-\Lambda^{2} y(n)=$ $g(n) y(n)+f(n) y(n+1)$, where $\Lambda$ is a large complex parameter, $a \geq 0$ and $g$ and $f$ are sequences of complex numbers. Two methods are proposed to find the asymptotic behavior for large $|\Lambda|$ of the solutions of this equation: (i) an iterative method based on a fixed point method and (ii) a discrete version of Olver's method for second-order linear differential equations. Both methods provide an asymptotic expansion of every solution of this equation. The expansion given by the first method is also convergent and may be applied to nonlinear problems. Bounds for the remainders are also given. We illustrate the accuracy of both methods for the modified Bessel functions and the associated Legendre functions of the first kind.
\end{abstract}

2010 AMS Mathematics Subject Classification: 39A06; 41A58; 41A60; 34B27.

Keywords \& Phrases: Second-order difference equations. Asymptotic expansions. Green's functions. Olver's method.

\section{Introduction}

Second-order difference equations are an important branch of mathematics with an active research and development. A comprehensive study of second-order difference equations and generalizations to higher order equations and partial difference equations may be found in classical references, like for example [13], [15] and [26], or in more modern references like [7] and [20]. In these references and in other related ones, we can find definitions, general properties, techniques of resolution in particular situations, stability properties, systems of difference equations, boundary value problems, eigenvalue problems and many other aspects of the theory of difference equations.

On the other hand, difference equations are an essential ingredient in mathematical models for discrete evolution problems that arise in different applied sciences such as biology, biochemistry, economy, etc., where the time variable is considered a discrete variable. Simple economical models that involve difference equations may be used to determine the evolution of the national income of a certain economy [13, Sec. 3.6, example 1], [25, Sec. 5.2]; to study inventory analysis [13, Sec. 3.6, example 3]; to analyze market price's behavior or Keynesian models with naive expectations $[17$. Chap. 1], multiplier-accelerator models for business cycles [17, Chap. 2], [10, Sec. 8.4], 25, Sec. 
5.1] or output, inflation and inflationary expectations [17, Chap. 3]; and to study interest and loan repayment [10, Sec. 8.2], models of supply and demand [10, Sec. 8.3], and chaos and competition in models of consumer behavior [25, Sec. 5.3]. There are also several biological systems analyzed by means of difference or systems of difference equations, like for example models of restricted or unrestricted population growth (discrete logistic equation, measles epidemic) 10 , Chap. 9]; models for population genetics (random mating, selection and mutation) [10, Chap. 10]. Other models of the economy and biological sciences may be found in 25 and references therein. Difference equations are also used to describe discrete dynamics in physical systems [26] and discrete hamiltonian systems (including discrete symplectic systems and discrete Jacobi equations) [1. Certain particular discrete dynamical models like population models, neural networks and replicator equations can be found in [2]. The use of difference equations to approximate the solutions of differential problems in field theory may be found in [27].

Difference equations are also important in several branches of mathematics: many sequences that occur in enumerative combinatorics satisfy a second-order linear difference equation [30; a great number of special functions of mathematical physics and, in particular, orthogonal polynomials, satisfy a three-term recurrence relation, which is a second-order linear difference equation [16. Sec 18.9]; the discretization of a second-order differential equation yields a second-order difference equation [14. Chaps. 3 and 4]; the coefficients of the Taylor expansion of the solutions of a differential equation obtained by the method of Frobenius are the solution of a difference equation. The behavior of the solutions of linear recurrence equations for large values of the discrete independent variable $n$ is important for combinatorics and computer science, numerical analysis, orthogonal polynomials, continued fractions and many other areas of pure and applied mathematics. It is also important in the applications mentioned in the previous paragraphs, as it is important to approximate the solution of the difference equations modeling the above mentioned problems for large $n$ (large time). A classical result in this topic are the theorems of Poincaré and Perron [12, Chap. 4].

On the other hand, in general, the difference equations of the economic, biological or physical models contain parameters and then, it is interesting to analyze the solutions of the equations for certain asymptotic values of one or several of their parameters. In particular, and as we show in Section 5, many special functions are solutions of difference equations with parameters, and it is interesting to approximate these functions in terms of simpler functions for certain asymptotic limits of those parameters. As we have mentioned in the previous paragraph, most of the investigations about the asymptotics of the solutions of linear difference equations are concerned with the behavior for large $n$ and fixed values of the parameters (see for example [3, 4, 11, 12, 21, 22, 28, 29,31] and references therein). But, as far as we know, the literature concerned with the behavior of the solutions for large values of the parameters is very limited. We can just mention the asymptotic technique proposed in [18] to approximate orthogonal polynomials in terms of simpler orthogonal polynomials in certain asymptotic limits of some of their parameters for fixed degree $n$; although the method is restricted to orthogonal polynomials of the Askey tableau and is not directly based on the difference equation satisfied by the polynomials. Then, in this paper we are interested in the asymptotic theory of difference equations for large values of the parameters and fixed $n$. More precisely, we study the asymptotic behavior of the solutions of linear second-order difference equations when one parameter is large and $n$ is fixed; we consider difference equations of the form

$$
y(n+2)-2 a y(n+1)-\Lambda^{2} y(n)=g(n) y(n)+f(n) y(n+1), \quad n=0,1,2, \ldots,
$$

where $\Lambda$ is a large complex parameter and $a \geq 0$. In this equation, $g$ and $f$ are sequences of complex numbers and of the order $\mathcal{O}(\Lambda)$ and $\mathcal{O}(1)$, respectively, as $|\Lambda| \rightarrow \infty$. Equation (1) is a particular case of the Sturn-Liouville difference equation considered in [15, Chap. 7], where $\Lambda^{2}$ represents the eigenvalue parameter. Therefore, the asymptotic methods introduced in this paper 
may be used to study the large eigenvalues of the Sturn-Liouville problem considered in 15 , Chap. 7].

The parameter a could be absorbed in the definition of $f(n)$ but, for convenience in the analysis, we consider equation (1) as it stands. For example, the case $a=1$ and $f(n)=0$ leads to the natural discretization of the second-order linear differential equation containing a large parameter considered by F. W. J. Olver in his asymptotic method for second-order linear differential equations without singular or turning points [23, Chap. 10]:

$$
y^{\prime \prime}=\left[\Lambda^{2}+g(x)\right] y, \quad x \in[0,+\infty),
$$

where $g$ is an analytic function.

Motivated by the analogy of (1) with its continuous counterpart (2), we propose in this paper two methods to approximate the solutions of (1) for large $|\Lambda|$ and fixed $n$. In the following section we consider the iterative method designed in [19] for an initial value problem related to the differential equation (2) and generalized in 8,9 ; we adapt this method to the difference equation (1), obtaining an asymptotic and convergent expansion of the unique solution of an initial value problem involving equation (1). In Section 3 we show that this iterative method may also be applied to nonlinear problems. In Section 4 we consider a different technique, the asymptotic method designed in [23. Chap. 10] for equation (2) when $|\Lambda|$ is large and $x$ is fixed; we adapt the method to its corresponding discrete formulation (1), obtaining the Poincaré-type asymptotic expansion of two independent solutions of (1). Finally, Section 5 contains two examples, the modified Bessel functions and the associated Legendre functions of the first kind, with numerical experiments confirming the validity of the two methods designed, and some concluding remarks.

\section{An iterative method}

In this section we are interested in the following initial value problem that selects one of the solutions of (1):

$$
\left\{\begin{array}{l}
y(n+2)-2 a y(n+1)-\Lambda^{2} y(n)=g(n) y(n)+f(n) y(n+1), \\
y(0)=\alpha, \quad y(1)=\beta,
\end{array}\right.
$$

with $\alpha, \beta \in \mathbb{C}$ and $\alpha=\mathcal{O}(1), \beta=\mathcal{O}(\Lambda)$ as $\Lambda \rightarrow \infty, n \in M:=\{0,1,2, \ldots, N\}, N \in \mathbb{N}$ fixed and $a^{2}+\Lambda^{2} \neq 0$.

For later convenience, we define, for $n \in M$ and $k=0,1,2, \ldots, n-1$, the sequences,

$$
G(n, k):=\frac{1}{2 \sqrt{a^{2}+\Lambda^{2}}}\left[\left(a+\sqrt{a^{2}+\Lambda^{2}}\right)^{n-k-1}-\left(a-\sqrt{a^{2}+\Lambda^{2}}\right)^{n-k-1}\right]
$$

and

$$
H(n):=\left(a+\sqrt{a^{2}+\Lambda^{2}}\right)^{n} .
$$

We use the symbol $\|\cdot\|_{\infty}$ as the usual supremum norm for bounded sequences $u(n)$ :

$$
\|u\|_{\infty}=\sup _{n \in M}|u(n)|
$$

We have the following theorem.

Theorem 1. Let $g$ and $f$ be two sequences of complex numbers. Then, for $m=0,1,2, \ldots$ and fixed $n \in M$, the sequence $y^{(m)}(n)$ obtained with the recursion

$$
\left\{\begin{array}{l}
y^{(m+1)}(n)=\phi(n)+\sum_{k=0}^{n-2} G(n, k)\left[g(k) y^{(m)}(k)+f(k) y^{(m)}(k+1)\right], \\
y^{(0)}(n)=\phi(n),
\end{array}\right.
$$


with

$$
\phi(n):=\frac{\alpha\left(\sqrt{a^{2}+\Lambda^{2}}-a\right)+\beta}{2 \sqrt{a^{2}+\Lambda^{2}}}\left(a+\sqrt{a^{2}+\Lambda^{2}}\right)^{n}+\frac{\alpha\left(\sqrt{a^{2}+\Lambda^{2}}+a\right)-\beta}{2 \sqrt{a^{2}+\Lambda^{2}}}\left(a-\sqrt{a^{2}+\Lambda^{2}}\right)^{n},
$$

converges to the unique solution $y(n)$ of problem (3). Moreover, it converges after a finite number of steps $m=n-1$.

Proof. The function $\phi(n)$ defined in $(8)$ is the unique solution of the auxiliary initial value problem

$$
\left\{\begin{array}{l}
\phi(n+2)-2 a \phi(n+1)-\Lambda^{2} \phi(n)=0 \\
\phi(0)=\alpha, \quad \phi(1)=\beta
\end{array}\right.
$$

Then, after the change of unknown $y(n) \rightarrow u(n):=y(n)-\phi(n)$, problem (3) reads

$$
\left\{\begin{array}{l}
u(n+2)-2 a u(n+1)-\Lambda^{2} u(n)=F(n, u):=g(n)[u(n)+\phi(n)]+f(n)[u(n+1)+\phi(n+1)], \\
u(0)=0, \quad u(1)=0 .
\end{array}\right.
$$

We seek solutions of the equation $\mathbf{L}[u]:=u(n+2)-2 a u(n+1)-\Lambda^{2} u(n)-F(n, u)=0$ in the Banach space of bounded finite sequences $\mathcal{B}_{0}=\left\{u(n), n \in M ;\|u\|_{\infty}<\infty, u(0)=u(1)=0\right\}$, equipped with the norm (6).

We write the equation $\mathbf{L}[u]=0$ as $\mathbf{L}[u]=\mathbf{M}[u]-F(n, u)$, with $\mathbf{M}[u]:=u(n+2)-2 a u(n+$ 1) $-\Lambda^{2} u(n)$. Then we solve the equation $\mathbf{L}[u]=0$ for $u$ in the form $u=\mathbf{M}^{-1}[F(n, u)]$, where $\mathbf{M}^{-1}$ is the inverse of the operator $\mathbf{M}$ in the space $\mathcal{B}_{0}$. For any $v \in \mathcal{B}_{0}$, that inverse is given by $\mathbf{M}^{-1}(v)=\sum_{k=0}^{\infty} \bar{G}(n, k) v(k)$, where $\bar{G}(n, k)$ is the corresponding Green's function of the problem $\mathbf{M}[u]=v$ with homogeneous conditions [15, Sec. 6.3]. That is, $\bar{G}(n, k)$ is the unique solution of the problem

$$
\left\{\begin{array}{l}
\bar{G}(n+2, k)-2 a \bar{G}(n+1, k)-\Lambda^{2} \bar{G}(n, k)=\delta_{n, k}, \\
\bar{G}(0, k)=\bar{G}(1, k)=0,
\end{array}\right.
$$

where $\delta_{n, k}=1$ if $n=k$ and $\delta_{n, k}=0$ if $n \neq k$. After a straightforward computation we obtain

$$
\bar{G}(n, k)=\left\{\begin{array}{ccc}
G(n, k) & \text { if } & n>k+1 \\
0 & \text { if } & n \leq k+1
\end{array}\right.
$$

with $G(n, k)$ defined in (4). Then, the solution $u(n)$ of $(9)$ is the solution of

$$
u(n)=\mathbf{M}^{-1}[F[n, u]]=\sum_{k=0}^{n-2} G(n, k)\{g(k)[u(k)+\phi(k)]+f(k)[u(k+1)+\phi(k+1)]\} .
$$

Or equivalently, the exact solution of problem (3) satisfies the equation

$$
y(n)=\phi(n)+\sum_{k=0}^{n-2} G(n, k)[g(k) y(k)+f(k) y(k+1)] .
$$

To prove the convergence of the recurrence (7) for a given $n \in M$ after a finite number of steps $m=n-1$, that is, $y^{(m)}(n)=y(n)$ for $n=0,1,2, \ldots, m+1$, and $m=0,1,2,3, \ldots$, we will use formula (11) and induction over $m$ :

- For $m=0$, we have that $y^{(0)}(n)=\phi(n)$ and then $y^{(0)}(0)=\phi(0)=y(0)$ and $y^{(0)}(1)=\phi(1)=$ $y(1)$. Then the hypothesis is true for $m=0$. 
- Suppose now that it is true for a given $m>0$. We know that $y^{(m)}(n)=y(n)$ for $n=$ $0,1,2,3, \ldots, m+1$. Consider any $n \leq m+2$ and subtract (7) and (11):

$$
y^{(m+1)}(n)-y(n)=\sum_{k=0}^{n-2} G(n, k)\left\{g(k)\left[y^{(m)}(k)-y(k)\right]+f(k)\left[y^{(m)}(k+1)-y(k+1)\right]\right\} .
$$

Using the induction hypothesis we have that, inside the brackets we find $y^{(m)}(k)-y(k)=0$ for any $k$ as $k$ runs from $k=0$ to $k=n-2 \leq m$ and $y^{(m)}(k+1)-y(k+1)=0$ for any $k$ as $k+1$ runs from $k+1=1$ to $k+1=n-1 \leq m+1$. Therefore,

$$
y^{(m+1)}(n)-y(n)=0
$$

for $n=0,1,2,3, \ldots, m+2$ and the thesis follows.

In the remaining of this section we show that the recursion (7) may be arranged in the form of an asymptotic expansion of $y(n)$ for large $|\Lambda|$. To this end we define a bounding constant $U$ and the remainder $R^{(m)}(n)$ in the form:

$$
U:=\frac{\|g\|_{\infty}}{\left|a+\sqrt{a^{2}+\Lambda^{2}}\right|}+\|f\|_{\infty}, \quad R^{(m)}(n):=H^{-1}(n)\left[y(n)-y^{(m)}(n)\right]
$$

where $y(n)$ is the solution of (3) and $y^{(m)}(n)$ is obtained from (7) after $m$ steps.

Theorem 2. Under the same hypothesis of Theorem 1, the remainder term (12) is bounded by

$$
\left|R^{(m)}(n)\right| \leq \frac{(n-1)_{m} U^{m}\left\|H^{-1} \cdot(y-\phi)\right\|_{\infty}}{\left|a^{2}+\Lambda^{2}\right|^{m / 2} m !} .
$$

Proof. In the proof of Theorem 1, we have shown that any solution of (9) can be written in the form 10. Equivalently, defining

$$
\tilde{u}(n):=H^{-1}(n) u(n) \quad \text { and } \quad \tilde{\phi}(n):=H^{-1}(n) \phi(n),
$$

with $H(n)$ and $\phi(n)$ given by (5) and (8) respectively, we have that for any solution $u(n)=$ $H(n) \tilde{u}(n)$ of $(9), \tilde{u}(n)$ is a solution of the equation

$$
\tilde{u}(n)=[\mathbf{T} \tilde{u}](n),
$$

where we have defined the operator $\mathbf{T}: \mathcal{B}_{0} \rightarrow \mathcal{B}_{0}$ :

$$
\begin{aligned}
& {[\mathbf{T} \tilde{u}](n):=\frac{1}{2 \sqrt{a^{2}+\Lambda^{2}}} \sum_{k=0}^{n-2} } {\left[1-\left(\frac{a-\sqrt{a^{2}+\Lambda^{2}}}{a+\sqrt{a^{2}+\Lambda^{2}}}\right)^{n-k-1}\right] } \\
& \times\left\{\frac{[\tilde{u}(k)+\tilde{\phi}(k)] g(k)}{a+\sqrt{a^{2}+\Lambda^{2}}}+[\tilde{u}(k+1)+\tilde{\phi}(k+1)] f(k)\right\} .
\end{aligned}
$$

It is clear that if we define

$$
\tilde{u}^{(k)}(n)=H^{-1}(n)\left[y^{(k)}(n)-\phi(n)\right], \quad k=0,1,2, \ldots,
$$


with $y^{(k)}$ given by $(7)$, then $\tilde{u}^{(m+1)}(n)=\left[\mathbf{T} \tilde{u}^{(m)}\right](n)$. Then, for any couple $z(n), w(n) \in \mathcal{B}_{0}$ :

$$
\begin{aligned}
& |[\mathbf{T} z](n)-[\mathbf{T} w](n)| \\
& \leq \frac{1}{2 \sqrt{\left|a^{2}+\Lambda^{2}\right|}} \sum_{k=0}^{n-2}\left|1-\left(\frac{a-\sqrt{a^{2}+\Lambda^{2}}}{a+\sqrt{a^{2}+\Lambda^{2}}}\right)^{n-k-1}\right| \\
& \quad \times\left[\frac{|z(k)-w(k)||g(k)|}{\left|a+\sqrt{a^{2}+\Lambda^{2}}\right|}+|z(k+1)-w(k+1)||f(k)|\right] \\
& \leq \frac{U}{2 \sqrt{\left|a^{2}+\Lambda^{2}\right|}}\|z-w\|_{\infty} \sum_{k=0}^{n-2}\left|1-\left(\frac{a-\sqrt{a^{2}+\Lambda^{2}}}{a+\sqrt{a^{2}+\Lambda^{2}}}\right)^{n-k-1}\right| \\
& \leq \frac{U}{2 \sqrt{\left|a^{2}+\Lambda^{2}\right|}}\|z-w\|_{\infty} \sum_{k=1}^{n-1}\left(1+\left|\frac{a-\sqrt{a^{2}+\Lambda^{2}}}{a+\sqrt{a^{2}+\Lambda^{2}}}\right|^{k}\right) \leq \frac{U(n-1)}{\sqrt{\left|a^{2}+\Lambda^{2}\right|}}\|z-w\|_{\infty} .
\end{aligned}
$$

We also have

$$
\begin{aligned}
& \left|\left[\mathbf{T}^{2} z\right](n)-\left[\mathbf{T}^{2} w\right](n)\right| \\
& \leq \frac{1}{2 \sqrt{\left|a^{2}+\Lambda^{2}\right|}} \sum_{k=0}^{n-2}\left|1-\left(\frac{a-\sqrt{a^{2}+\Lambda^{2}}}{a+\sqrt{a^{2}+\Lambda^{2}}}\right)^{n-k-1}\right| \\
& \times\left[\frac{|[\mathbf{T} z](k)-[\mathbf{T} w](k)||g(k)|}{\left|a+\sqrt{a^{2}+\Lambda^{2}}\right|}+|[\mathbf{T} z](k+1)-[\mathbf{T} w](k+1) \| f(k)|\right] \\
& \leq \frac{U^{2}}{2\left|a^{2}+\Lambda^{2}\right|}\|z-w\|_{\infty} \sum_{k=1}^{n-1}\left(1+\left|\frac{a-\sqrt{a^{2}+\Lambda^{2}}}{a+\sqrt{a^{2}+\Lambda^{2}}}\right|^{k}\right) k \leq \frac{U^{2}(n-1) n}{2\left|a^{2}+\Lambda^{2}\right|}\|z-w\|_{\infty} .
\end{aligned}
$$

It is straightforward to prove, by means of induction over $m$, that for $m=1,2,3, \ldots$

$$
\left|\left[\mathbf{T}^{m} z\right](n)-\left[\mathbf{T}^{m} w\right](n)\right| \leq \frac{(n-1) n(n+1)(n+2) \ldots(n+m-2)}{\left|a^{2}+\Lambda^{2}\right|^{m / 2} m !} U^{m}\|z-w\|_{\infty},
$$

and then

$$
\left\|\mathbf{T}^{m} z-\mathbf{T}^{m} w\right\|_{\infty} \leq \frac{U^{m}(n-1)_{m}}{\left|a^{2}+\Lambda^{2}\right|^{m / 2} m !}\|z-w\|_{\infty},
$$

where $(n)_{m}$ denotes the Pochhammer symbol: $(n)_{m}:=n(n+1)(n+2) \ldots(n+m-1)$.

To prove bound (13), we set $z=\tilde{u}$ and $w=\tilde{u}^{(0)}=0$ in (17). Using that $\mathbf{T}^{m} \tilde{u}=\tilde{u}$ and $T^{m} \tilde{u}^{(0)}=\tilde{u}^{(m)}$ we find

$$
\left|\tilde{u}(n)-\tilde{u}^{(m)}\right| \leq \frac{U^{m}(n-1)_{m}}{\left|a^{2}+\Lambda^{2}\right|^{m / 2} m !}\|\tilde{u}\|_{\infty} .
$$

We have seen in Theorem 1 that the unique solution $y(n)$ of problem (3) may be obtained from the limit $y(n)=\lim _{m \rightarrow \infty} y^{(m)}(n)$, where $y^{(m)}(n)$ is the recurrence relation defined in (7) and (8). (Indeed we have that $y^{(m)}(n)=y(n)$ for $m \geq n-1$.) In other words, $y(n)$ admits the series expansion

$$
y(n)=\phi(n)+\sum_{k=0}^{n-2}\left[y^{(k+1)}(n)-y^{(k)}(n)\right]=\phi(n)+H(n) \sum_{k=0}^{n-2}\left[\tilde{u}^{(k+1)}(n)-\tilde{u}^{(k)}(n)\right],
$$


with $\tilde{u}^{(k)}(n)$ defined in $(16)$. Using the definition $(12)$ for the remainder $R^{(m)}(n)$ we find that, for $m<n-1$, we may write $(19)$ in the form

$$
\begin{aligned}
y(n) & =\phi(n)+\sum_{k=0}^{m-1}\left[y^{(k+1)}(n)-y^{(k)}(n)\right]+H(n) R^{(m)}(n) \\
& =\phi(n)+H(n)\left[\sum_{k=0}^{m-1}\left[\tilde{u}^{(k+1)}(n)-\tilde{u}^{(k)}(n)\right]+R^{(m)}(n)\right] .
\end{aligned}
$$

In the next theorem we analyze the asymptotic properties of this expansion.

Theorem 3. Let the functions $y^{(m)}(n)$ be defined by the recurrence relation (7) and (8) with $g=\mathcal{O}(\Lambda)$ and $f$ of the order $\mathcal{O}(1)$ when $|\Lambda| \rightarrow \infty$. Then, for $n \in M$, the expansion (20) is an asymptotic expansion for large $|\Lambda|$ of the unique solution of $(3)$. More precisely, for $m=$ $0,1,2,3, \ldots$,

$$
\tilde{u}^{(m)}(n)-\tilde{u}^{(m-1)}(n)=\mathcal{O}\left(\Lambda^{-m}\right) \quad \text { and } \quad R^{(m)}(n)=\mathcal{O}\left(\Lambda^{-m-1}\right) .
$$

Proof. From definition 15 we have

$$
\begin{aligned}
\tilde{u}^{(m)}(n)=\left[\mathbf{T} \tilde{u}^{(m-1)}\right](n) & =\frac{1}{2 \sqrt{a^{2}+\Lambda^{2}}} \sum_{k=0}^{n-2}\left[1-\left(\frac{a-\sqrt{a^{2}+\Lambda^{2}}}{a+\sqrt{a^{2}+\Lambda^{2}}}\right)^{n-k-1}\right] \\
& \times\left\{\frac{\left[\tilde{u}^{(m-1)}(k)+\tilde{\phi}(k)\right] g(k)}{a+\sqrt{a^{2}+\Lambda^{2}}}+\left[\tilde{u}^{(m-1)}(k+1)+\tilde{\phi}(k+1)\right] f(k)\right\}
\end{aligned}
$$

and

$$
\begin{aligned}
\tilde{u}^{(m+1)}(n)= & {\left[\mathbf{T} \tilde{u}^{(m)}\right](n) } \\
=\frac{1}{2 \sqrt{a^{2}+\Lambda^{2}}} \sum_{k=0}^{n-2} & {\left[1-\left(\frac{a-\sqrt{a^{2}+\Lambda^{2}}}{a+\sqrt{a^{2}+\Lambda^{2}}}\right)^{n-k-1}\right] } \\
\times & \left\{\frac{\left[\tilde{u}^{(m)}(k)+\tilde{\phi}(k)\right] g(k)}{a+\sqrt{a^{2}+\Lambda^{2}}}+\left[\tilde{u}^{(m)}(k+1)+\tilde{\phi}(k+1)\right] f(k)\right\} .
\end{aligned}
$$

Subtracting 22 and 23 we get

$$
\left\|\tilde{u}^{(m+1)}-\tilde{u}^{(m)}\right\|_{\infty} \leq \frac{U(n-1)}{\sqrt{\left|a^{2}+\Lambda^{2}\right|}}\left\|\tilde{u}^{(m)}-\tilde{u}^{(m-1)}\right\|_{\infty} .
$$

We have $\tilde{u}^{(0)}(n)=0$ and $\tilde{u}^{(1)}(n)=\left[T \tilde{u}^{(0)}\right](n)=\mathcal{O}\left(\Lambda^{-1}\right)$ for $n \in M$. Using this and (24), the first thesis in (21) follows by induction over $m$.

Setting $z=\tilde{u}$ and $w=\tilde{u}^{(0)}=0$ in the inequality (18) (as we did in the proof of Theorem 2), we find

$$
\left\|\tilde{u}-\tilde{u}^{(m)}\right\|_{\infty} \leq \frac{U^{m}(n-1)_{m}}{\left|a^{2}+\Lambda^{2}\right|^{m / 2} m !}\|\tilde{u}\|_{\infty} .
$$

But $\tilde{u}=\lim _{m \rightarrow \infty} \tilde{u}^{(m)}=\sum_{k=0}^{n-2}\left[\tilde{u}^{(k+1)}-\tilde{u}^{(k)}\right]=\sum_{k=0}^{n-2} \mathcal{O}\left(\Lambda^{-k-1}\right)=\mathcal{O}\left(\Lambda^{-1}\right)$ for $n \in M$. This and inequality (25) prove the second thesis in (21).

\footnotetext{
${ }^{1}$ Indeed, this expression is also valid for $m \geq n-1$, although trivial, as $R^{(m)}(n)=0$ for $m \geq n-1$.
} 


\section{The nonlinear case}

The iterative method introduced in Section 2 can be generalized to approximate the solution of nonlinear second-order difference problems of the form

$$
\left\{\begin{array}{l}
y(n+2)-2 a y(n+1)-\Lambda^{2} y(n)=h(n, y(n), y(n+1)), \\
y(0)=\alpha, \quad y(1)=\beta
\end{array}\right.
$$

where $h$ is continuous in its three variables, $a \geq 0, \Lambda$ is a large complex parameter and $\alpha=\mathcal{O}(1)$, $\beta=\mathcal{O}(\Lambda)$. We also require for the forthcoming analysis that the function $h$ satisfy a Lipchitz condition in its second and third variables:

$$
|h(n, u, v)-h(n, z, w)| \leq \Lambda K_{1}|u-z|+K_{2}|v-w|,
$$

fo any $u, v, z, w \in \mathbb{C}, n \in M$ and $K_{1}$ and $K_{2}$ positive constants independent of $n, u, v, z, w, \Lambda$. As in the linear case, we assume that $\Lambda^{2}+a^{2} \neq 0$. Now, in this nonlinear case, we assume the extra condition 2

$$
\bar{U}:=\frac{K_{1} \Lambda}{\left|a+\sqrt{a^{2}+\Lambda^{2}}\right|}+K_{2}<\sqrt{\left|a^{2}+\Lambda^{2}\right|} .
$$

We have the following theorem.

Theorem 4. Let $h$ be a continuous function that satisfies (27) and assume that (28) holds and $n \in M$ is fixed. Then,

1. For $m=0,1,2, \ldots$, the sequence $y^{(m)}(n)$ obtained with the recursion

$$
\left\{\begin{array}{l}
y^{(m+1)}(n)=\phi(n)+\sum_{k=0}^{n-2} G(n, k) h\left(k, y^{(m)}(k), y^{(m)}(k+1)\right), \\
y^{(0)}(n)=\phi(n),
\end{array}\right.
$$

with $G(n, k)$ given in (4) and $\phi(n)$ given by (8), converges to the unique solution $y(n)$ of (26): $\lim _{m \rightarrow \infty} y^{(m)}(n)=y(n)$.

2. The remainder $R^{(m)}(n)$ defined, as in the linear case, by formula 112 , is bounded by

$$
\left|R^{(m)}(n)\right| \leq \frac{(n-1)_{m} \bar{U}^{m}}{\left|a^{2}+\Lambda^{2}\right|^{m / 2} m !}\left\|H^{-1}(y-\phi)\right\|_{\infty} .
$$

Proof. We cannot prove the convergence $y^{(m)}(n) \rightarrow y(n)$ as in Theorem 1, as the first line in $(29)$ is nonlinear. But we can repeat step by step the proof of Theorem 2 , replacing $\|f\|_{\infty}$ and $\|g\|_{\infty}$ by $K_{1} \Lambda$ and $K_{2}$ respectively and the constant $U$ by the constant $\bar{U}$, to derive formula (30). From this formula it is clear that, under assumption (28), recursion (29) converges to the unique solution of (26): $\lim _{m \rightarrow \infty} y^{(m)}(n)=y(n)$.

Observe that now, we cannot assure that the convergence of the sequence $y^{(m)}(n) \rightarrow y(n)$ occurs after a finite number of steps, as it happens in the linear case. But, as well as in the linear case, the sequence $y^{(m)}(n)$ may be rearranged in the form of an asymptotic sequence of $y(n)$ for large $|\Lambda|$, as we show below. As in the linear case, the unique solution of (26) admits the series expansion (20) with $y^{(m)}(n), \tilde{u}^{(m)}(n)$ and $R^{(m)}(n)$ given by (29), (16) and (12) respectively. Then, we have the following theorem.

\footnotetext{
${ }^{2}$ This condition is not very restrictive whenever we are considering that the parameter $|\Lambda|$ is large.
} 
Theorem 5. Let the functions $y^{(m)}(n)$ be defined by the recurrence relation $(29)$ and (8) with $h$ a continuous function in its three variables that satisfies (27) and (28). Then, for $n \in M$, the expansion (20) is an asymptotic expansion for large $|\Lambda|$ of the unique solution of (26). More precisely, for $m=1,2,3, \ldots$,

$$
\tilde{u}^{(m)}(n)-\tilde{u}^{(m-1)}(n)=\mathcal{O}\left(\Lambda^{-m}\right) \quad \text { and } \quad R^{(m)}(n)=\mathcal{O}\left(\Lambda^{-m-1}\right) .
$$

Proof. Similar to the proof of Theorem 3 replacing $\|f\|_{\infty}$ and $\|g\|_{\infty}$ by $K_{1} \Lambda$ and $K_{2}$ respectively and $U$ by $\bar{U}$.

\section{Olver's method for problem (1)}

In Section 2 we have proposed a convergent and asymptotic iterative technique to approximate the solution of the initial value problem (3) for a linear difference equation. The method is based on its continuous counterpart [19 designed for differential equations. In Section 3 we have generalized the technique to the initial value problem $(26)$ for a nonlinear difference equation. In this section we propose a different technique to approximate the solution of (3), an asymptotic technique based on its continuous counterpart designed by Olver [23, Chap. 10] for differential equations. This method is not convergent in general.

We can summarize the discussion in the following theorem.

Theorem 6. Let $g$ and $f$ be two sequences of complex numbers. Then, for $m=0,1,2, \ldots$, fixed $n \in M$ and $|\Lambda| \rightarrow \infty$, the unique solution $y(n)$ of problem (3) admits the asymptotic expansion

$$
\begin{aligned}
y(n) & =\frac{\beta\left(\sqrt{a^{2}+\Lambda^{2}}+a\right)+\alpha \Lambda^{2}}{2 \sqrt{a^{2}+\Lambda^{2}}}\left(a+\sqrt{a^{2}+\Lambda^{2}}\right)^{n} \sum_{k=0}^{m-1} \frac{A(n, k)}{\left(a+\sqrt{a^{2}+\Lambda^{2}}\right)^{k}} \\
& +\frac{\beta\left(\sqrt{a^{2}+\Lambda^{2}}-a\right)-\alpha \Lambda^{2}}{2 \sqrt{a^{2}+\Lambda^{2}}}\left(a-\sqrt{a^{2}+\Lambda^{2}}\right)^{n} \sum_{k=0}^{m-1} \frac{A(n, k)}{\left(a-\sqrt{a^{2}+\Lambda^{2}}\right)^{k}}+\mathcal{O}\left(\Lambda^{n-m}\right),
\end{aligned}
$$

with $A(n, 0)=0, A(n, 1)=1$ and the remaining coefficients $A(n, k)$ are obtained from the recursion

$$
\begin{gathered}
A(n, k+2)=\sum_{j=1}^{\lfloor n / 2\rfloor}[g(n-2 j) A(n-2 j, k)+f(n-2 j) A(n+1-2 j, k+1) \\
+2 a A(n+1-2 j, k+1)-2 a A(n-2 j, k+1)] .
\end{gathered}
$$

Proof. Reproducing Olver's idea for differential equations, we consider two (at this moment unknown) independent solutions $y_{+}(n)$ and $y_{-}(n)$ of (1) and propose the following representations in the form of formal asymptotic expansions for large $|\Lambda|$ :

$$
y_{+}(n)=y_{+}^{(m)}(n)+R_{+}^{(m)}(n), \quad y_{-}(n)=y_{-}^{(m)}(n)+R_{-}^{(m)}(n),
$$

with

$$
y_{ \pm}^{(m)}(n):=\left(a \pm \sqrt{a^{2}+\Lambda^{2}}\right)^{n} \sum_{k=0}^{m-1} \frac{A(n, k)}{\left(a \pm \sqrt{a^{2}+\Lambda^{2}}\right)^{k}},
$$

and at this moment unknown coefficients $A(n, k)$ and remainders $R_{ \pm}^{(m)}(n)$. When we introduce (34) and (35) in the equation (1) we find that both, $y_{+}(n)$ and $y_{-}(n)$, formally satisfy this equation, 
term-wise in $\left(a+\sqrt{a^{2}+\Lambda^{2}}\right)^{k}$ and $\left(a-\sqrt{a^{2}+\Lambda^{2}}\right)^{k}$ respectively if, for $k=0,1,2, \ldots$, the following identities hold true:

$$
\left\{\begin{aligned}
A(n, 0)=0, \quad A(n, 1) & =1, \\
A(n+2, k+2)-2 a A & (n+1, k+1)-A(n, k+2)+2 a A(n, k+1) \\
& =g(n) A(n, k)+f(n) A(n+1, k+1) .
\end{aligned}\right.
$$

It is straightforward to show that we can solve this recursion in the variable $k$ in the form (33). Now, using that $y_{+}(n)$ and $y_{-}(n)$ satisfy equation (1), we find that the remainder terms $R_{+}^{(m)}(n)$ and $R_{-}^{(m)}(n)$ in (34) must be the solutions of the respective problems:

$$
\left\{\begin{aligned}
R_{ \pm}^{(m)}(n+2)-2 a R_{ \pm}^{(m)}(n+1)-\Lambda^{2} R_{ \pm}^{(m)}(n)= & g(n)\left(R_{ \pm}^{(m)}(n)+\frac{A(n, m-1)}{\left(a \pm \sqrt{a^{2}+\Lambda^{2}}\right)^{m-n-1}}\right) \\
& +f(n) R_{ \pm}^{(m)}(n+1)+\frac{A(n+2, m)-A(n, m)}{\left(a \pm \sqrt{a^{2}+\Lambda^{2}}\right)^{m-n-2}} \\
R_{ \pm}^{(m)}(0)=0, \quad R_{ \pm}^{(m)}(1)=0, &
\end{aligned}\right.
$$

These problems for $R_{ \pm}^{(m)}(n)$ are identical to problem (9) for $u(n)$ replacing $g(n) \phi(n)+f(n) \phi(n+1)$ by

$$
g(n) \frac{A(n, m-1)}{\left(a \pm \sqrt{a^{2}+\Lambda^{2}}\right)^{m-n-1}}+\frac{A(n+2, m)-A(n, m)}{\left(a \pm \sqrt{a^{2}+\Lambda^{2}}\right)^{m-n-2}} .
$$

Therefore, proceeding as in the proof of Theorem 1 , we find that $R_{+}^{(m)}(n)$ and $R_{-}^{(m)}(n)$ satisfy an equation similar to 10$)$ :

$$
\begin{aligned}
R_{ \pm}^{(m)}(n)= & \sum_{k=0}^{n-2} G(n, k)\left[g(k) R_{ \pm}^{(m)}(k)+f(k) R_{ \pm}^{(m)}(k+1)\right] \\
& +\sum_{k=0}^{n-2} G(n, k)\left[g(k) \frac{A(k, m-1)}{\left(a \pm \sqrt{a^{2}+\Lambda^{2}}\right)^{m-k-1}}+\frac{A(k+2, m)-A(k, m)}{\left(a \pm \sqrt{a^{2}+\Lambda^{2}}\right)^{m-k-2}}\right] .
\end{aligned}
$$

We define now the normalized remainder:

$$
\tilde{R}_{ \pm}^{(m)}(n):=\left(a \pm \sqrt{a^{2}+\Lambda^{2}}\right)^{-n} R_{ \pm}^{(m)}(n)
$$

Then, we have that

$$
\begin{aligned}
& \tilde{R}_{+}^{(m)}(n)= \frac{1}{2 \sqrt{a^{2}+\Lambda^{2}}} \sum_{k=0}^{n-2}\left[1-\left(\frac{a-\sqrt{a^{2}+\Lambda^{2}}}{a+\sqrt{a^{2}+\Lambda^{2}}}\right)^{n-k-1}\right]\left[\frac{g(k) \tilde{R}_{+}^{(m)}(k)}{a+\sqrt{a^{2}+\Lambda^{2}}}+f(k) \tilde{R}_{+}^{(m)}(k+1)\right] \\
&+\frac{1}{2 \sqrt{a^{2}+\Lambda^{2}}} \sum_{k=0}^{n-2}\left[1-\left(\frac{a-\sqrt{a^{2}+\Lambda^{2}}}{a+\sqrt{a^{2}+\Lambda^{2}}}\right)^{n-k-1}\right] \\
& \times\left[g(k) \frac{A(k, m-1)}{\left(a+\sqrt{a^{2}+\Lambda^{2}}\right)^{m}}+\frac{A(k+2, m)-A(k, m)}{\left(a+\sqrt{a^{2}+\Lambda^{2}}\right)^{m-1}}\right] .
\end{aligned}
$$


Taking bounds we find

$$
\begin{aligned}
\left|\tilde{R}_{+}^{(m)}(n)\right| \leq & \frac{1}{2 \sqrt{\left|a^{2}+\Lambda^{2}\right|}} \sum_{k=0}^{n-2}\left|1-\left(\frac{a-\sqrt{a^{2}+\Lambda^{2}}}{a+\sqrt{a^{2}+\Lambda^{2}}}\right)^{n-k-1}\right|\left[\frac{|g(k)|\left|\tilde{R}_{+}^{(m)}(k)\right|}{\left|a+\sqrt{a^{2}+\Lambda^{2}}\right|}+|f(k)|\left|\tilde{R}_{+}^{(m)}(k+1)\right|\right] \\
& +\frac{1}{2 \sqrt{\left|a^{2}+\Lambda^{2}\right|}} \sum_{k=0}^{n-2}\left|1-\left(\frac{a-\sqrt{a^{2}+\Lambda^{2}}}{a+\sqrt{a^{2}+\Lambda^{2}}}\right)^{n-k-1}\right|\left[|g(k)| \frac{|A(k, m-1)|}{\left|a+\sqrt{a^{2}+\Lambda^{2}}\right|^{m}}\right. \\
& \left.+\frac{|A(k+2, m)-A(k, m)|}{\left|a+\sqrt{a^{2}+\Lambda^{2}}\right|^{m-1}}\right]
\end{aligned}
$$

and

$$
\begin{aligned}
\left|\tilde{R}_{+}^{(m)}(n)\right| \leq & \frac{1}{\sqrt{\left|a^{2}+\Lambda^{2}\right|}} \sum_{k=0}^{n-2}\left[\frac{|g(k)|\left|\tilde{R}_{+}^{(m)}(k)\right|}{\mid a+\sqrt{a^{2}+\Lambda^{2} \mid}}+|f(k)|\left|\tilde{R}_{+}^{(m)}(k+1)\right|\right] \\
& +\frac{1}{\sqrt{\left|a^{2}+\Lambda^{2}\right|}} \sum_{k=0}^{n-2}\left[\frac{|g(k)||A(k, m-1)|}{\left|a+\sqrt{a^{2}+\Lambda^{2}}\right|^{m}}+\frac{|A(k+2, m)-A(k, m)|}{\left|a+\sqrt{a^{2}+\Lambda^{2}}\right|^{m-1}}\right] .
\end{aligned}
$$

Defining now

$$
h_{1}(k):=\frac{1}{\sqrt{\left|a^{2}+\Lambda^{2}\right|}} \begin{cases}\frac{|g(0)|}{\left|a+\sqrt{a^{2}+\Lambda^{2}}\right|}, & \text { if } k=0, \\ \frac{|g(k)|}{\left|a+\sqrt{a^{2}+\Lambda^{2}}\right|}+|f(k-1)|, & \text { if } 1 \leq k \leq n-2, \\ |f(n-2)|, & \text { if } k=n-1,\end{cases}
$$

and

$$
\begin{aligned}
h_{2}(n):= & \frac{\|g\|_{\infty}}{\sqrt{\left|a^{2}+\Lambda^{2}\right|\left|a+\sqrt{a^{2}+\Lambda^{2}}\right|^{m}} \sum_{k=0}^{n-2}|A(k, m-1)|} \\
& +\frac{1}{\sqrt{\left|a^{2}+\Lambda^{2}\right|}\left|a+\sqrt{a^{2}+\Lambda^{2}}\right|^{m-1}} \sum_{k=0}^{n-2}|A(k+2, m)-A(k, m)|,
\end{aligned}
$$

we can rewrite bound 36 as

$$
\left|\tilde{R}_{+}^{(m)}(n)\right| \leq h_{2}(n)+\sum_{k=0}^{n-1} h_{1}(k)\left|\tilde{R}_{+}^{(m)}(k)\right| .
$$

Applying discrete Gronwall's Lemma [5], we find

$$
\left|\tilde{R}_{+}^{(m)}(n)\right| \leq h_{2}(n)+\sum_{k=0}^{n-1} h_{2}(k) h_{1}(k) e^{\sum_{j=k+1}^{n-1} h_{1}(j)} .
$$

Thus, $\tilde{R}_{+}^{(m)}(n)=\mathcal{O}\left(\Lambda^{-m}\right)$, and then $R_{+}^{(m)}(n)=\left(a+\sqrt{a^{2}+\Lambda^{2}}\right)^{n} \tilde{R}_{+}^{(m)}(n)=\mathcal{O}\left(\Lambda^{n-m}\right)$. Similarly, we obtain that $R_{-}^{(m)}(n)=\mathcal{O}\left(\Lambda^{n-m}\right)$. These bounds show the asymptotic character of the expansions $y_{ \pm}(n) \sim y_{ \pm}^{(m)}(n)$ given in $\left.(34)-35\right)$.

The unique solution $y(n)$ of problem (3) is a certain linear combination of the two independent solutions $y_{+}(n)$ and $y_{-}(n): y(n)=A y_{+}(n)+B y_{-}(n)$, for certain constants $A$ and $B$. Therefore, 
the unique solution $y(n)$ of problem (3) may be approximated, up to order $\mathcal{O}\left(\Lambda^{n-m}\right)$, by that linear combination replacing $y_{ \pm}(n)$ by their respective asymptotic approximations $y_{ \pm}^{(m)}(n)$. That is, we have that $y(n)=y^{(m)}(n)+\mathcal{O}\left(\Lambda^{n-m}\right)$, with $y^{(m)}(n):=A y_{+}^{(m)}(n)+B y_{-}^{(m)}(n)$. We compute the approximate value (up to order $\mathcal{O}\left(\Lambda^{n-m}\right)$ ) of the constants $A$ and $B$, by imposing the conditions $y^{(m)}(0)=\alpha$ and $y^{(m)}(1)=\beta$, obtaining

$$
A=\frac{\beta\left(\sqrt{a^{2}+\Lambda^{2}}+a\right)+\alpha \Lambda^{2}}{2 \sqrt{a^{2}+\Lambda^{2}}}, \quad B=\frac{\beta\left(\sqrt{a^{2}+\Lambda^{2}}-a\right)-\alpha \Lambda^{2}}{2 \sqrt{a^{2}+\Lambda^{2}}},
$$

from where $(32)$ follows.

\section{$5 \quad$ Examples}

In this section we apply the two techniques proposed in the respective sections 2 and 4 for the approximation of the solution of the second-order difference problem (3) to the particular examples of the modified Bessel functions and the associated Legendre functions of the first kind.

Example 1. Consider the second-order difference equation

$$
\left\{\begin{array}{l}
y(n+2)+\frac{2(n+1)}{z} y(n+1)-y(n)=0, \quad n=0,1,2, \ldots, \\
y(0)=1, \quad y(1)=I_{1}(z) / I_{0}(z)
\end{array}\right.
$$

where $z \in \mathbb{C}$ and $I_{0}(z)$ and $I_{1}(z)$ are modified Bessel functions and whose solution is $y(n)=$ $I_{n}(z) / I_{0}(z)$ (see [24, Formula 10.6.1]). After the change of dependent variable $y(n)=u(n) / z^{n}$, (38) can be written in the form

$$
\left\{\begin{array}{l}
u(n+2)-z^{2} u(n)=-2(n+1) u(n+1), \\
u(0)=1, \quad u(1)=z I_{1}(z) / I_{0}(z)
\end{array}\right.
$$

This problem is of the form of equation (1) with $a=0, \Lambda^{2}=z^{2}, g(n)=0, f(n)=-2(n+1)$, $\alpha=1$ and $\beta=z I_{1}(z) / I_{0}(z)$. The exact solution of $(39)$ is given by $u(n)=z^{n} I_{n}(z) / I_{0}(z)$.

Then, the iterative method introduced in Section 2 provides a convergent as well as an asymptotic expansion of $I_{n}(z)$ for large $|z|$ and fixed $n$ given by the recurrence relation (7):

$$
u^{(0)}(n)=\phi(n)= \begin{cases}z^{n}, & \text { if } n \text { is even, } \\ z^{n} I_{1}(z) / I_{0}(z), & \text { if } n \text { is odd. }\end{cases}
$$

and, for $m=0,1,2, \ldots$,

$$
u^{(m+1)}(n)=\phi(n)-\sum_{k=0}^{n-2}\left[1-(-1)^{n-k-1}\right](k+1) z^{n-k-2} u^{(m)}(k+1) .
$$

On the other hand, using Olver's method given in Section 4 , we find that $I_{n}(z)$ admits, for large $z$ and fixed $n$, the asymptotic expansion $(32), u(n)=u^{(m)}(n)+\mathcal{O}\left(\Lambda^{n-m}\right)$, with

$$
u^{(m)}(n)=\frac{I_{1}(z)+I_{0}(z)}{2 I_{0}(z)} \sum_{k=0}^{m-1} \frac{A(n, k)}{z^{k-n-1}}+\frac{I_{1}(z)-I_{0}(z)}{2 I_{0}(z)} \sum_{k=0}^{m-1} \frac{A(n, k)}{(-z)^{k-n-1}}
$$

where $A(n, 0)=0, A(n, 1)=1$ and

$$
A(n, k+2)=-2 \sum_{j=1}^{\lfloor n / 2\rfloor}(n+1-2 j) A(n+1-2 j, k+1), \quad k \geq 0 .
$$


Table 1 shows the asymptotic character of both methods, 40 and (41), in the approximation of the terms $u(8)$ and $u(10)$ of the solution of problem $(39)$ for different values of $\Lambda$ and using the approximation given by $m=5$ and $m=6$ respectively.

\begin{tabular}{|c|c|c|}
\hline$z$ & Method (40) & Method (41) \\
\hline$-9+100 i$ & 0.00001388 & 0.00001388 \\
\hline$-9+1000 i$ & $1.38 e-10$ & $1.38 e-10$ \\
\hline$-9+10000 i$ & $1.38 e-15$ & $1.38 e-15$ \\
\hline
\end{tabular}

\begin{tabular}{|c|c|c|}
\hline$z$ & Method $(40)$ & Method $(41)$ \\
\hline 100 & 0.00001425 & 0.00001425 \\
\hline 1000 & $9.45 e-12$ & $9.45 e-12$ \\
\hline 10000 & $9.10 e-18$ & $9.10 e-18$ \\
\hline
\end{tabular}

Table 1: Relative errors in the approximation of the terms $u(8)$ and $u(10)$ in problem (39) using the iterative method (40) and Olver's method (41) for different values of $\Lambda$ and for $m=5$ and $m=6$ respectively. The table shows the asymptotic character of both methods.

Example 2. Consider the second-order difference equation

$$
\left\{\begin{array}{l}
y(n+2)+\frac{2(n+1) z}{\sqrt{z^{2}-1}} y(n+1)+\left[\left(n+\frac{1}{2}\right)^{2}-\tau^{2}\right] y(n)=0, \quad n=0,1,2, \ldots \\
y(0)=1, \quad y(1)=P_{\tau-1 / 2}^{1}(z) / P_{\tau-1 / 2}^{0}(z)
\end{array}\right.
$$

where $z, \tau \in \mathbb{C}$ and $P_{\tau-1 / 2}^{0}(z)$ and $P_{\tau-1 / 2}^{1}(z)$ are associated Legendre functions of the first kind. The unique solution of this problem is $y(n)=P_{\tau-1 / 2}^{n}(z) / P_{\tau-1 / 2}^{0}(z)$ (see [6, Formula 14.10.6]).

This problem is of the type (1) with $a=0, \Lambda=\tau, g(n)=-\left(n+\frac{1}{2}\right)^{2}, f(n)=-\frac{2(n+1) z}{\sqrt{z^{2}-1}}, \alpha=1$ and $\beta=P_{\tau-1 / 2}^{1}(z) / P_{\tau-1 / 2}^{0}(z)$. The exact solution of 442$)$ is $y(n)=P_{\tau-1 / 2}^{n}(z) / P_{\tau-1 / 2}^{0}(z)$.

Then, the iterative method introduced in Section 2 provides a convergent as well as an asymptotic expansion of $P_{\tau-1 / 2}^{n}(z)$ for large $|\tau|$ and fixed $n$ given by the recurrence relation (7):

$$
y^{(0)}(n)=\phi(n)=\frac{P_{\tau-1 / 2}^{0}(z) \tau+P_{\tau-1 / 2}^{1}(z)}{2 \tau P_{\tau-1 / 2}^{0}(z)} \tau^{n}+\frac{P_{\tau-1 / 2}^{0}(z) \tau-P_{\tau-1 / 2}^{1}(z)}{2 \tau P_{\tau-1 / 2}^{0}(z)}(-\tau)^{n} .
$$

and, for $m=0,1,2, \ldots$,

$$
y^{(m+1)}(n)=\phi(n)-\frac{1}{2 \tau} \sum_{k=0}^{n-2}\left[\tau^{n-k-1}-(-\tau)^{n-k-1}\right]\left[\left(k+\frac{1}{2}\right)^{2} y^{(m)}(k)+\frac{2(k+1) z}{\sqrt{z^{2}-1}} y^{(m)}(k+1)\right] .
$$

On the other hand, using Olver's method given in Section 4, we have that $P_{\tau-1 / 2}^{n}(z)$ can be approximated, for large $\tau$ and fixed $n$, by the expansion (32): $y(n)=y^{(m)}(n)+\mathcal{O}\left(\Lambda^{n-m}\right)$, with

$$
y^{(m)}(n)=\frac{P_{\tau-1 / 2}^{1}(z)+P_{\tau-1 / 2}^{0}(z) \tau}{2 P_{\tau-1 / 2}^{0}(z)} \tau^{n} \sum_{k=0}^{m-1} \frac{A(n, k)}{\tau^{k}}+\frac{P_{\tau-1 / 2}^{1}(z)-P_{\tau-1 / 2}^{0}(z) \tau}{2 P_{\tau-1 / 2}^{0}(z)}(-\tau)^{n} \sum_{k=0}^{m-1} \frac{A(n, k)}{(-\tau)^{k}},
$$

where $A(n, 0)=0, A(n, 1)=1$ and

$$
A(n, k+2)=-\sum_{j=1}^{\lfloor n / 2\rfloor}\left[\left(n-2 j+\frac{1}{2}\right)^{2} A(n-2 j, k)+\frac{2(n+1-2 j) z}{\sqrt{z^{2}-1}} A(n+1-2 j, k+1)\right], k \geq 0 .
$$


Table 2 shows the asymptotic character of the two methods, (43) and (44), in the approximation of the term $y(5)$ for $z=2$ of the solution of problem (42) for different values of $\tau$ and using the approximation given by $m=4$.

\begin{tabular}{|c|c|c|}
\hline$\tau$ & Method $(43)$ & Method (44) \\
\hline 5 & 0.31558587 & 0.13986491 \\
\hline 50 & 0.00011022 & 0.00004879 \\
\hline 500 & $1.09 e-8$ & $4.83 e-9$ \\
\hline
\end{tabular}

\begin{tabular}{|c|c|c|}
\hline$\tau$ & Method $\sqrt[43]{ })$ & Method $(44)$ \\
\hline $5 e^{i \pi / 4}$ & 0.23446498 & 0.10377073 \\
\hline $50 e^{i \pi / 4}$ & 0.00009004 & 0.00003290 \\
\hline $500 e^{i \pi / 4}$ & $1.07 e-8$ & $3.12 e-9$ \\
\hline
\end{tabular}

Table 2: Relative errors in the approximation of the term $y(5)$ of problem (42) using the iterative method (43) and Olver's method (44) for different values of $\tau, z=2$ and $m=4$. The table shows the asymptotic character of both methods.

\section{$6 \quad$ Final remarks}

From the first example of the previous section we may suspect that the approximations given by both, the iterative method and Olver's method, are exactly the same ones (at any order $m$ ). In fact, this is true and is not genuine of this example, but a general property for any difference equation with $a=0$ and $g(n)=0$ : under these circumstances, the iterative method (7) reads

$$
y^{(m+1)}(n)=\phi(n)+\sum_{k=1}^{\lfloor n / 2\rfloor} \Lambda^{2 k-2} f(n-2 k) y^{(m)}(n+1-2 k), \quad y^{(0)}(0)=0,
$$

with

$$
\phi(n)=\frac{\alpha \Lambda+\beta}{2 \Lambda} \Lambda^{n}+\frac{\alpha \Lambda-\beta}{2 \Lambda}(-\Lambda)^{n} .
$$

On the other hand, Olver's approximation 32 reads

$$
y^{(m)}(n)=\sum_{k=0}^{m-1}\left[\frac{\alpha \Lambda+\beta}{2 \Lambda} \Lambda^{n-k+1}+\frac{\alpha \Lambda-\beta}{2 \Lambda}(-\Lambda)^{n-k+1}\right] A(n, k)=\sum_{k=0}^{m-1} \phi(n-k+1) A(n, k),
$$

with

$$
A(n, k+2)=\sum_{j=1}^{\lfloor n / 2\rfloor} f(n-2 j) A(n+1-2 j, k+1),
$$

and $A(n, 0)=0, A(n, 1)=1$. It can be checked that, Olver's approximation (47) satisfies the recurrence relation (45) and (46) if and only if the coefficients $A(n, k)$ in (47) verify the equation (48). Thus, for $a=0$ and $g(n)=0$, the iterative method and Olver's method provide the same expansion and we can deduce that, in this case, Olver's method is not only asymptotic but also convergent.

In the continuous case (Olver's theory [23, Chap. 10] for linear second order differential equations), the two coefficients $A$ and $B$ of the linear combination of the two approximate independent solutions must be computed at every step of the approximation an then, they depend on the order $m$ of the approximation. The situation is very different in the discrete case because, as we see in (37), the coefficients $A$ and $B$ of the linear combination that gives the approximate solution of problem $(3): y^{(m)}(n)=A y_{+}^{(m)}(n)+B y_{-}^{(m)}(n)$, are independent of the degree $m$ of the approximation. Once they are computed for a certain order $m$ of the approximation, they are computed for any order. 


\section{Acknowledgments}

This research was supported by the Spanish Ministry of "Economia y Competitividad", project MTM2014-52859-P. The Universidad Pública de Navarra is acknowledged by its financial support.

\section{References}

[1] C. D. Ahlbrandt And A. C. Peterson, Discrete Hamiltonian Systems, Springer-Science+Busnisess Media, B.V., 1996.

[2] Z. Alsharawi, J. M. Cushing And S. Elaydi Eds., Theory and Applications of Difference Equations and Discrete Dynamical Systems, Springer Proceedings in Mathematics and Statistics, Springer, New York, 2013.

[3] L. Cao And Y. Li, Linear difference equations with a transition point at the origin, Anal. Appl. (Singap.), 12 (2014), no. 1, 75-106.

[4] J. R. CASH, An extension of Olver's method for the numerical solution of linear recurrence relations, Mathematics of Computation, 32 (1978), no. 2, 497-510.

[5] J. Dieudonné, Foundations of Modern Analysis, Academic Press, New York, 1960.

[6] T. M. Dunster, Legendre and Related Functions, in NIST Handbook of Mathematical Functions, U.S. Department of Commerce, Washington, DC, 2010. Available online at http://dlmf.nist.gov/14.

[7] S. Elaydi, An Introduction to Difference Equations, Springer, New York, 2005.

[8] C. Ferreira, J. L. López and E. PÉrez Sinusía, Convergent and asymptotic expansions of solutions of differential equations with a large parameter: Olver cases II and III, J. Integral Equations Appl., 27 (2015), no. $1,27-45$.

[9] C. Ferreira, J. L. López and E. PÉrez Sinusía, Convergent and asymptotic expansions of solutions of second-order differential equations with a large parameter, Anal. Appl. (Singap.), 12 (2014), no. 5, 523-536.

[10] G. Fulford, P. Forrester And A. Jones, Modeling with Differential and Difference Equations, Cambridge Univ. Press, New York, 1997.

[11] J. S. Geronimo and D. T. Smith, WKB (Liouville-Green) analysis of second order difference equations and applications, J. Approx. Theory, 69 (1992), no. 3, 269-301.

[12] A. Gil, J. Segura and N. M. Temme, Numerical Methods for Special Functions, SIAM, Philadelphia, 2007.

[13] S. Goldberg, Introduction to Difference Equations, Dover, New York, 1986.

[14] B. S. Jovanović, Analysis of Finite Difference Schemes, Springer, London, 2014.

[15] W. G. Kelley And A. C. Peterson, Difference Equations. An Introduction with Applications, Academic Press, New York, 1991.

[16] T. H. Koornwinder, R. Wong, R. Koekoek and R. F. Swarttouw, Orthogonal Polynomials, in NiSt Handbook of Mathematical Functions, U.S. Department of Commerce, Washington, DC, 2010. Available online at http://dlmf.nist.gov/10.

[17] A. Levy, Economic Dynamics, Avebury, Hong Kong, 1992.

[18] J.L. López AND N. Temme, Approximations of orthogonal polynomials in term of Hermite polynomials, Meth. Appl. Anal., 6 (1999), no. 2, 131-146.

[19] J. L. López, Olver's asymptotic method revisited. Case I, J. Math. Anal. Appl., 395 (2012), no. 2, 578-586.

[20] R. E. Mickens, Difference Equations. Theory, Applications and Advanced Topics, CRC Press, New York, 2015.

[21] F. W. J. Olver, Bounds for the solutions of second-order linear difference equations, TJ. Res. Nat. Bur. Standards Sect. B, 71B (1967), 111-129.

[22] F. W. J. Olver, Numerical solution of second-order linear difference equations, TJ. Res. Nat. Bur. Standards Sect. B, 71B (1967), 161-166.

[23] F. W. J. Olver, Asymptotics and Special Functions, Academic Press, New York, 1974.

[24] F. W. J. Olver And L. C. Maximon, Bessel Functions, in NIST Handbook of Mathematical Functions, U.S. Department of Commerce, Washington, DC, 2010. Available online at http://dlmf.nist.gov/10.

[25] H. Sedaghat, Nonlinear Difference Equations. Theory with Applications to Social Science Models, SpringerScience+Busnisess Media, B.V., 2003. 
[26] A. N. Sharkovsky, Y. L. Maistrenko and E. Yu Romanenko, Difference Equations and their Applications, Springer, The Netherlands, 1993.

[27] A. J. Schwab, Field Theory Concepts, Springer-Verlag, New York, 1988.

[28] R. Spigler AND M. Vianello, Liouville-Green approximations for a class of linear oscillatory difference equations of the second order, J. Comput. Appl. Math., 41 (1992), no. 1-2, 105-116.

[29] R. Spigler, M. Vianello And F. Locatelli, Liouville-Green-Olver approximations for complex difference equations, J. Approx. Theory, 96 (1999), no. 2, 301-322.

[30] R. P. Stanley, Enumerative Combinatorics, Vol. 1, Cambridge University Press, New York, 2012.

[31] R. Wong, Asymptotics of linear recurrences., Anal. Appl. (Singap.), 12 (2014), no. 4, 463-484. 